\title{
Role of Montelukast as Monotherapy in Improving Quality of Life of an Asthmatic Patient in Pakistan
}

\author{
Ankeeta Kumari ${ }^{1}$, Mishal Ejaz ${ }^{2}$, Khurram Anis $^{3}$, Sumeet Kumar ${ }^{4}$, Besham Kumar $^{5}$, Sidra Memon ${ }^{6}$ \\ 1. Medicine, Liaquat University of Medical and Health Sciences, Jamshoro, PAK 2. Internal Medicine, Ziauddin \\ University, Karachi, PAK 3. Gastroenterology/Hepatology, Pakistan Kidney and Liver Institute, Lahore, PAK 4. Internal \\ Medicine, Chandka Medical College Hospital, Larkana, PAK 5. Internal Medicine, Jinnah Postgraduate Medical Centre, \\ Karachi, PAK 6. Internal Medicine, Jinnah Sindh Medical University, Karachi, PAK
}

Corresponding author: Besham Kumar, beshamkumar916@gmail.com

\begin{abstract}
Introduction: Uncontrolled and inadequately managed asthma substantially reduces Quality of Life (QOL) and can lead to premature death. The aim of this study is to understand the role of montelukast in improving quality of life in asthmatic patients by comparing it with placebo.
\end{abstract}

Methods: This prospective, single-arm, interventional study was conducted from September 2019 to February 2020 in the pulmonology outpatient department of a tertiary care hospital in Pakistan. All patients were prescribed montelukast (10 mg once daily).

Results: At day 28, participants had a higher score on the Asthma Quality of Life Questionnaire-Standard (AQLQ-S) overall and in all sub-domains compared to day 0 . The improvement was significant overall and for the sub-domains of symptoms, activity limitations, and environmental stimuli.

Conclusion: Montelukast has an effective role in asthma control as well as in improving the quality of life in the Pakistani population.

Categories: Pulmonology

Keywords: montelukast, pakistan, quality of life

\section{Introduction}

Asthma is characterized by an airway reaction leading to reversible airflow obstruction in association with airway hyper-responsiveness (AHR) and airway inflammation [1]. Asthma is quite common worldwide affecting approximately 339 million people, and according to studies, it is estimated to affect 100 million more people by the year 2025 [2]. When comparing geographical and demographic data, it is variable. Studies have suggested that high-income countries of the world have more people suffering from asthma than in average or low-income countries [3]. The report on global burden of asthma estimated the prevalence of asthma in Pakistan to be 4.3\% [4]. Despite being quite common, it is not adequately being managed, leading to low Quality of Life (QOL) and early death. Asthma is rated as the 16th cause of Disability Adjusted Life Years (DALYs). Asthma also leads to increased burden of disease, as it ranks 28th among other causes [2].

Management of asthma is crucial. Inhaled corticosteroids (ICS) prove to produce promising results, however leukotriene receptor antagonists (LTRAs) are becoming more and more popular these days due to feasibility of use. LTRAs in contrast to ICS do not predispose the patient to long-term adverse effects [5]. LTRAs have been recommended by Global Initiative for Asthma (GINA) guidelines to be used as a single agent for asthma, can be used as an add-on or substitute to ICS increasing doses, or long-acting $\beta 2$-agonist (LABA) [6].

In Pakistan, fewer studies have been conducted that study the role of montelukast in the management of asthma. Hence our aim is to study its role to help pulmonologists manage asthma effectively by reducing the morbidity and mortality of the disease.

\section{Materials And Methods}

This prospective, single-arm, interventional study was conducted from September 2019 to February 2020 in the pulmonology outpatient department of a tertiary care hospital in Pakistan. Both male and female asthmatic patients who were older than 15 years were enrolled in this study. Patients who presented with acute exacerbations of asthma and those who had previously taken montelukast were excluded from the study. All patients were given montelukast (10 mg once daily). Compliant patients were defined as those who took montelukast for at least 21 days. The patients were instructed to bring their strips on follow-up to 


\section{Cureus}

ensure compliance.

A total of 156 participants were enrolled and were followed for four weeks. Patients who weren't followed-up were not included in the analysis. A self-structured questionnaire was used to record the participant's age, gender, duration of asthma, and symptoms. Quality of life was evaluated using the Asthma Quality of Life Questionnaire-Standard (AQLQ-S) on day 0 and day 60. AQLQ-S has 32 items assessing four domains: symptoms, activity, emotions, and environment control. A higher score means a higher quality of life [7].

Data were processed through and analyzed using SPSS for Windows version 23.0 (IBM Corp., Armonk, NY, USA). AQLQ-S mean and standard deviation (SD) were calculated for continuous variables such as age and AQLQ-S. Frequency and percentage were calculated for categorical variables including gender, age, duration of asthma, and symptoms. A comparison was done for scores of AQLQ-S on days 0 and 60 using dependent T-test. P-values of less than 0.05 meant there is a difference between AQLQ-S score on day 0 and day 60 and that the null hypothesis is void.

\section{Results}

At the start of the study, 156 asthmatic patients were included. During the study, one (0.6\%) patient stopped taking montelukast due to an adverse event and 21 (13.4\%) participants were not followed-up. The study was completed by 134 (85.9\%) participants, who were included in the final analysis. All these participants were compliant. The mean age of participants included in the analysis was $22 \pm 8$ years, of which 68 (50.7\%) participants were men and 66 (49.3\%) were women. Their disease characteristics are shown in Table 1

\begin{tabular}{|l|l|}
\hline Patient Characteristics & Frequency (\%) (n=134) \\
\hline Duration of Asthma & $15(11.1 \%)$ \\
\hline$<1$ year & $47(35.0 \%)$ \\
\hline 3-6 years & $38(25.8 \%)$ \\
$\geq 7$ years & $34(25.3 \%)$ \\
\hline Symptoms & $105(78.3 \%)$ \\
Cough & $99(73.8 \%)$ \\
Wheezing & $88(65.6 \%)$ \\
Chest Tightness & $97(72.3 \%)$ \\
Shortness of Breath & \\
\hline
\end{tabular}

TABLE 1: Patient Characteristics

At day 28, participants had a higher score on the Asthma Quality of Life Questionnaire-Standard (AQLQ-S) overall and in all sub-domains compared to day 0 . The improvement was significant overall and for the subdomains of symptoms, activity limitations, and environmental stimuli (Table 2). 


\section{Cureus}

\begin{tabular}{|c|c|c|c|c|}
\hline AQLQ-S & At Day 0 (Mean \pm SD) & At Day 28 (Mean \pm SD) & Difference & P value* \\
\hline Overall & $4.12 \pm 0.62$ & $4.89 \pm 0.89$ & 0.77 & $<0.0001^{\star}$ \\
\hline Symptoms & $3.79 \pm 0.75$ & $4.18 \pm 0.37$ & 0.39 & $<0.0001^{\star}$ \\
\hline Activity Limitation & $4.41 \pm 0.99$ & $5.11 \pm 0.93$ & 0.70 & $<0.0001^{\star}$ \\
\hline Emotional Function & $4.42 \pm 1.20$ & $4.52 \pm 1.13$ & 0.10 & 0.48 \\
\hline Environmental Function & $4.59 \pm 1.03$ & $5.38 \pm 1.21$ & 0.79 & $<0.0001^{*}$ \\
\hline \multicolumn{5}{|c|}{ TABLE 2: AQLQ-S at Day 0 and Day 28} \\
\hline \multicolumn{5}{|c|}{ Abbreviations: AQLQ-S, Asthma Quality of Life Questionnaire - Standard; SD, standard deviation; } \\
\hline 'Dependent sample I-test & ween groups; $\mathrm{p}$ value $<0$ & ant. & & \\
\hline
\end{tabular}

\section{Discussion}

Montelukast is a leukotriene receptor antagonist. It reduces leukotriene D4 (LTD4) levels in the respiratory tract by competetively binding to the cysteinyl leukotriene receptor (CysLT1). LTD4 is a potent bronchoconstrictor and CysLT1 attracts inflammatory cells. Normally, the binding of CysLT1 with LTD4 leads to allergic and hypersensitivity reactions, therefore montelukast plays a vital role in the management of asthmatic patients [8].

In this study, there was significant improvement in the Asthma Quality of Life Questionnaire-Standard (AQLQ-S) overall. Among sub-domains, all domains had improved significantly except emotional function. The result is comparable to previous studies. Baig et al. in their double-blind trial compared montelukast with placebo. They found overall significant quality of life improvement, like our study; however, in their study only the environmental stimuli sub-domain of AQLQ-S had significant improvement compared to placebo [8]. Studies have suggested that montelukast, when given as an add-on therapy with ICS, can significantly improve QOL in asthma patients [9]. Other studies have also reported similar results, especially a study conducted by Virchow and his colleagues, who reported better morbidity as explained by mini-AQLQ scores, when montelukast was used as an adjunct to LABA and ICS [10]. Moreover, the same researcher in his other study investigated the efficacy of $10 \mathrm{mg}$ montelukast in adults with asthma in a phase IV clinical trial, conducted in different centres, and reported that there was an improvement in symptomatology of asthma. Around $92 \%$ of patients were satisfied with montelukast and considered continuing montelukast treatment over other asthma medications, and 85\% of patients suffering from asthma marked "very good" or "good" in the questionnaire [11].

In an experimental, double-blind, crossover study, researchers gave $20 \mathrm{mg}$ of montelukast two times a day for almost a week to a group of patients and compared the results with the patients taking placebo. They reported that the symptoms produced by leukotriene E4 (LTE4) were significantly improved in the patients taking montelukast [12]. This study resonates with the findings of another research by Bozek et al. who concluded that montelukast when added to a short-acting beta-agonist (SABA) resulted in fewer attacks of exacerbations of asthma [13]. However, there have been contrasting studies as well, which report the opposite. Columbo showed that there was insignificant improvement in asthma with montelukast when given to elderly patients after comparing Asthma Control Test (ACT) scores and measuring biochemical assay at the fourth and eighth week of therapy [14].

Nevertheless, there are various studies that support montelukast in the management of acute exacerbation of asthma as well as chronic asthma of varying severity. Montelukast ameliorates inflammation of the respiratory tract, as well as improves the overall symptomatology of the asthmatic patients. From various data available and our research on this topic, we can safely conclude that pulmonologists should consider using montelukast in the management of asthma as an add-on therapy with ICS or LABA to improve the overall morbidity and quality of life in asthma patients. Other studies should be done to compare if montelukast can replace ICS or not, as ICS has some long term side-effects. More studies are required to study the effect of montelukast on the mortality of asthma patients.

\section{Conclusions}

In this study, there was significant improvement in overall Asthma Quality of Life Questionnaire-Standard (AQLQ-S). Among sub-domains, environment functions, symptoms, and activity limitations had significant improvement. It is important to consider the impact on quality of life of various treatment options available for asthma. Our study, along with various global studies, shows that montelukast is effective in improving patients quality of life. 


\section{Additional Information}

Disclosures

Human subjects: Consent was obtained by all participants in this study. Liaquat University of Medical \& Health Sciences issued approval LUMHS/18/05C3. Animal subjects: All authors have confirmed that this study did not involve animal subjects or tissue. Conflicts of interest: In compliance with the ICMJE uniform disclosure form, all authors declare the following: Payment/services info: All authors have declared that no financial support was received from any organization for the submitted work. Financial relationships: All authors have declared that they have no financial relationships at present or within the previous three years with any organizations that might have an interest in the submitted work. Other relationships: All authors have declared that there are no other relationships or activities that could appear to have influenced the submitted work.

\section{References}

1. Holgate S: Innate and adaptive immune responses in asthma . Nat Med. 2012, 18:673-83. 10.1038/nm.2731

2. The Global Asthma Report 2018. (2018). http://www.globalasthmareport.org/.

3. To T, Stanojevic S, Moores G, et al.: Global asthma prevalence in adults: findings from the cross-sectional world health survey. BMC Public Health. 2012, 12:5. 10.1186/1471-2458-12-204

4. Masoli M, Fabian D, Holt S, Beasley R: The global burden of asthma: executive summary of the GINA dissemination committee report. Allergy. 2004, 59:469-78. 10.1186/s12890-018-0753-y

5. Miligkos M, Bannuru RR, Alkofide H, Kher SR, Schmid CH, Balk EM: Leukotriene-receptor antagonists versus placebo in the treatment of asthma in adults and adolescents:a systematic review and meta-analysis. Ann Intern Med. 2015, 163:756-767. 10.7326/M15-1059

6. Global Initiative for Asthma (GINA) . (2018). https://ginasthma.org/2018-gina-report-global-strategy-forasthma-management-and-prevention/.

7. Juniper EF, Buist AS, Cox FM, Ferrie PJ, King DR: Validation of a standardized version of the asthma quality of life questionnaire. Chest. 1999, 115:1265-70. 10.1378/chest.115.5.1265

8. Baig S, Khan RA, Khan K, Rizvi N: Effectiveness and quality of life with montelukast in asthma - a doubleblind randomized control trial. Pak J Med Sci. 2019, 35:731-736. 10.12669/pjms.35.3.42

9. Biernacki WA, Kharitonov SA, Biernacka HM, Barnes PJ: Effect of montelukast on exhaled leukotrienes and quality of life in asthmatic patients. Chest. 2005, 128:1958-63. 10.1378/chest.128.4.1958

10. Virchow JC, Mehta A, Ljungblad L, Mitfessel H. MONICA Study Group: Add-on montelukast in inadequately controlled asthma patients in a 6-month open-label study: the MONtelukast In Chronic Asthma (MONICA) study. Respir Med. 2010, 104:644-51. 10.1016/j.rmed.2009.11.022

11. Virchow JC, Bachert C: Efficacy and safety of montelukast in adults with asthma and allergic rhinitis . Respir Med. 2006, 100:1952-1959. 10.1016/j.rmed.2006.02.026

12. Lazarinis N, Bood J, Wheelock C, Dahlen SE, Dahlen B: The CysLT1receptor antagonist montelukast blocks bronchoconstriction induced by inhaled leukotriene E4 in subjects with asthma. Eur Respir J. 2015, 46:2545. 10.1183/13993003.congress-2015.PA2545

13. Bozek A, Warkocka-Szoltysek B, Filipowska-Gronska A, Jarzab J: Montelukast as an add-on therapy to inhaled corticosteroids in the treatment of severe asthma in elderly patients. J Asthma. 2012, 49:530-534. 10.3109/02770903.2012.680638

14. Columbo M: Asthma in the elderly: a double-blind, placebo-controlled study of the effect of montelukast . Asthma Res Pract. 2017, 3:3. 10.1186/s40733-017-0031-4 